$$
\begin{array}{r}
\text { Pontifícia Universidade Católica } \\
\text { do Rio de Janeiro }
\end{array}
$$

Fábio Bicalho de Araújo

\title{
Impacto da Geração Distribuída nas Condições de Estabilidade de Tensão
}

Dissertação de Mestrado

Dissertação apresentada como requisito parcial para obtenção do grau de Mestre pelo Programa de Pósgraduação em Engenharia Elétrica do Departamento de Engenharia Elétrica da PUC-Rio.

Orientador: Prof. Ricardo Bernardo Prada

Rio de Janeiro

Dezembro de 2010 


$$
\text { Pontifícia } \text { Universidade }_{\text {do Rio de Janeiro }}
$$

Fábio Bicalho de Araujo

\begin{abstract}
Impacto da Geração Distribuída nas Condições de Estabilidade de Tensão
\end{abstract}

\begin{abstract}
Dissertação apresentada como requisito parcial para obtenção do grau de Mestre pelo Programa de PósGraduação em Engenharia Elétrica do Departamento de Engenharia Elétrica do Centro Técnico Científico da PUCRio. Aprovada pela Comissão Examinadora abaixo assinada.
\end{abstract}

Prof. Ricardo Bernardo Prada

Orientador

Departamento de Engenharia Elétrica - PUC-Rio

Prof. Walmir de Freitas Filho Universidade Estadual de Campinas

Prof. Jorgeson Oliveira Rodrigues dos Santos Universidade Federal de São João Del Rei

Prof. José Eugenio Leal

Coordenador Setorial do Centro Técnico Científico - PUC-Rio

Rio de Janeiro, 16 de dezembro de 2010 
Todos os direitos reservados. É proibida a reprodução total ou parcial do trabalho sem autorização da universidade, do autor e do orientador.

\section{Fábio Bicalho de Araújo}

Ex-aluno do Colégio Pedro II e técnico em Telecomunicações pelo CEFET/RJ graduou-se em Engenharia Elétrica pela Pontifícia Universidade Católica do Rio de Janeiro, PUC-Rio. Durante a graduação foi membro do Conselho de Ensino e Pesquisa da PUC-Rio, bolsista de iniciação científica pelo CNPq, monitor de cálculo I, além de outras atividades acadêmicas. Atualmente, dedica-se a atividades voltadas para o mercado de engenharia elétrica e ao setor elétrico.

Araújo, Fábio Bicalho de

Impacto da geração distribuída nas condições de estabilidade de tensão / Fábio Bicalho de Araújo ; orientador: Ricardo Bernardo Prada. - 2010.

147 f. ; $30 \mathrm{~cm}$

Dissertação (mestrado) - Pontifícia Universidade Católica do Rio de Janeiro, Departamento de Engenharia Elétrica, 2010.

Inclui bibliografia

1. Engenharia elétrica - Teses. 2. Segurança de tensão. 3. Estabilidade de tensão. 4. Controle de tensão. 5. Colapso de tensão. 6. Barras de tensão controlada. 7. Geração distribuída. 8. Compensador síncrono. 9. Fluxo de potência. 10. Sistemas de distribuição. I. Prada, Ricardo Bernardo. II. Pontifícia Universidade Católica do Rio de Janeiro. Departamento de Engenharia Elétrica. III. Título. 
Dedico este trabalho à minha mãe, Nádia Santoro Bicalho. Em especial, dedico aos meus avôs maternos Ciro e Magda Bicalho e paternos, Mario e Ruth Barros de Araujo. Obrigado por tudo! Sem vocês não seria possível este sonho se realizar. 


\section{Agradecimentos}

Sou eternamente grato à Pontifícia Universidade Católica do Rio de Janeiro pela maneira como fui acolhido ao iniciar o curso de graduação e posteriormente na pós-graduação. Sua política de bolsas de estudo para alunos carentes foi essencial para a minha formação como engenheiro. Não tenho palavras para descrever o orgulho que sinto por ter estudado e ter feito parte desta universidade. Sua contribuição para o ensino, pesquisa e para sociedade como um todo, é inestimável.

Gostaria de agradecer pela orientação e paciência despendidas pelo professor Ricardo B. Prada. Sua ajuda foi essencial para a consolidação dos meus conhecimentos de engenharia, sistematização e domínio do tema escolhido. Sou-lhe eternamente grato pela oportunidade de desenvolver esta dissertação e pelas perspectivas que se abrem após a conclusão deste trabalho.

Um espaço muito especial deve ser reservado à minha família. Instituição pela qual devo todos os agradecimentos possíveis que possam existir neste mundo. Todos foram, são, e continuarão sendo muito importantes nesta jornada e na minha vida.

Finalmente, agradeço aos meus amigos e a todas as outras pessoas que contribuíram de alguma forma para o êxito deste trabalho: principalmente aos amigos, funcionários e pesquisadores do Laboratório de Sistemas de Energia Elétrica da PUC-Rio e de todo o Campus.

Agradeço ao CEPEL pelo uso do programa ANAREDE, à CAPES e à FAPERJ pelas bolsas de estudo.

Neste exato momento em que escrevo, relembrando de todas as dificuldades, alegrias, medos, e felicidades que tive nestes oito anos de graduação e pósgraduação, me sinto emocionado. Valeu o esforço. Muito obrigado! 


\section{Resumo}

Araújo, Fábio Bicalho de; Prada, Ricardo Bernardo (Orientador). Impacto da Geração Distribuída nas Condições de Estabilidade de Tensão. Rio de Janeiro, 2010. 147p. Dissertação de Mestrado - Departamento de Engenharia Elétrica. Pontifícia Universidade Católica do Rio de Janeiro.

Após a incidência de inúmeros colapsos de tensão em sistemas elétricos de potência por todo o mundo, a segurança de tensão tornou-se um assunto de extrema importância. O fenômeno de estabilidade de tensão deve-se à operação do sistema com carregamento elevado e se manifesta pela existência de uma máxima transferência de potência pela rede e ações de controle de tensão tendo efeito oposto ao usual. Com o aumento da inserção de geração distribuída, usualmente conectada à rede de distribuição existente e em tensões mais baixas, a possibilidade de ocorrência do fenômeno precisa ser investigada. É demonstrada a possibilidade de ocorrência em barras com geradores ou compensadores síncronos, onde a máxima potência gerada poderia estar limitada e o controle de tensão poderia ter o efeito oposto ao esperado. É apresentado o ferramental utilizado na avaliação das condições de estabilidade de tensão composto de índices com significado físico e matemático. Estabeleceu-se um procedimento para realizar simulações nos sistemas-teste IEEE 34 Barras e 70 Barras. É analisado o impacto do gerador nas condições de estabilidade de tensão, no perfil de tensão em regime permanente e nas perdas de potência ativa. Concluiu-se que, nos testes realizados, a injeção de potência ativa pelo gerador foi limitada por tensões fora da faixa permitida e não por problemas de estabilidade de tensão.

\section{Palavras chave}

Segurança de tensão, estabilidade de tensão, controle de tensão, colapso de tensão, barras de tensão controlada, geração distribuída, compensador síncrono, fluxo de potência, sistemas de distribuição. 


\section{Abstract}

Araújo, Fábio Bicalho de; Prada, Ricardo Bernardo (Advisor). Distributed Generation Impact on Voltage Stability Conditions. Rio de Janeiro, 2010. 147p. MSc Dissertation - Departamento de Engenharia Elétrica. Pontifícia Universidade Católica do Rio de Janeiro.

After several incidence of voltage collapse in electric power systems around the world, the voltage security has become a matter of utmost importance. The phenomenon of voltage stability is due to the operation of the system with high loading and is manifested by the existence of a maximum power transfer over the network and efforts to control voltage having the opposite effect than usual. With increased integration of distributed generation, usually connected to the existing distribution network at lower voltages, the possibility of occurrence of the phenomenon should be investigated. It is demonstrated the possibility of occurrence in buses of synchronous generators or synchronous condensers, where the maximum generated power could be limited and the voltage control could have the opposite effect. It presented the tools used in assessing the conditions of voltage stability composite indexes with physical and mathematical meaning. I was established a procedure for performing simulations on IEEE 34 bus and 70 bus test systems. It analyzed the impact of the generator under the conditions of voltage stability, voltage profile in the steady state and the power losses. It was concluded that, in tests, the injection of active power from the generator was limited by voltage out of range allowed and not by voltage stability problems.

\section{Keywords}

Voltage security, voltage stability, voltage control, voltage colapse, voltage controlled bus, distributed generation, synchronous condenser, power flow, distribution systems. 


\section{Sumário}

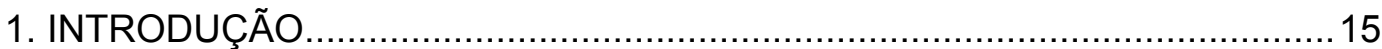

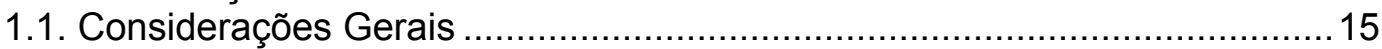

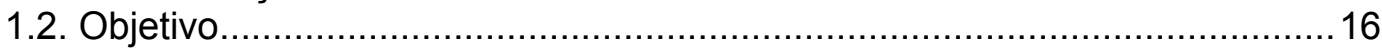

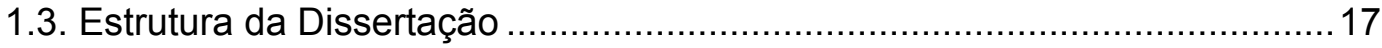

2. INTRODUÇÃO AO FENÔMENO DE ESTABILIDADE DE TENSÃO .............20

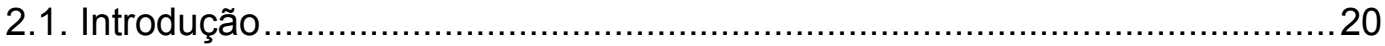

2.2. Caracterização do Fenômeno de Estabilidade de Tensão...........................21

2.2.1. Análise Gráfica do Fenômeno .............................................................22

2.2.2. Impedância da Carga no Máximo Carregamento .....................................22

2.2.3. Limite de Estabilidade de Tensão - LET ................................................... 31

2.2.4. Capacitor em Paralelo na Barra de Carga ............................................... 34

2.2.5. Exemplo da Saturação da Elevação da Tensão e do Aumento da

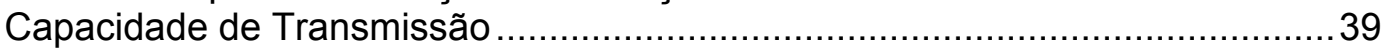

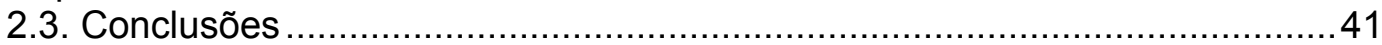

3. ESTUDO DA BARRA DE GERAÇÃO [1] ………….............................42

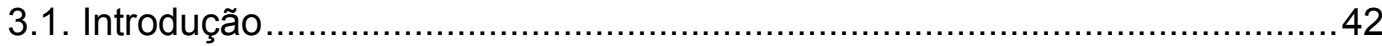

3.2. Potência Ativa e Reativa Saindo da Barra de Geração...............................42

3.3. Tensão Crítica na Barra de Geração........................................................4 44

3.4. Avaliação das Condições de Estabilidade de Barras de Tensão

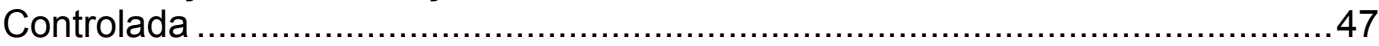

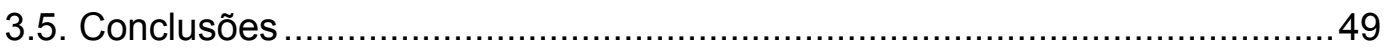

4. AVALIAÇÃO DAS CONDIÇÕES DE ESTABILIDADE DE TENSÃO [7] [8].....51

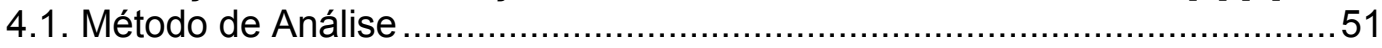

4.2. Magnitude do Determinante da Matriz [D'] ................................................ 53

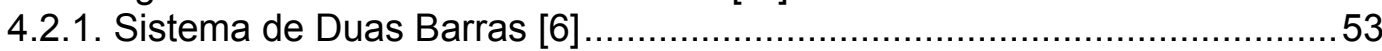

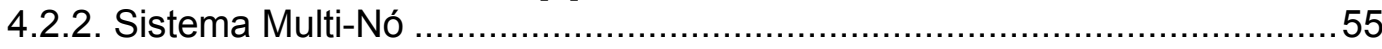

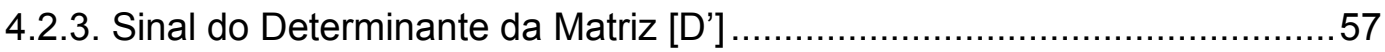

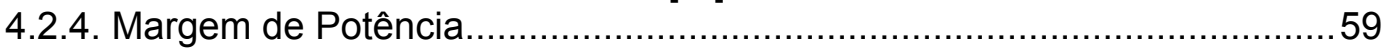

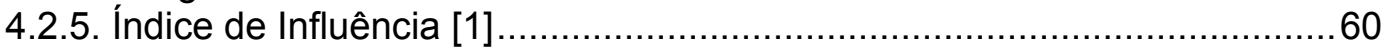

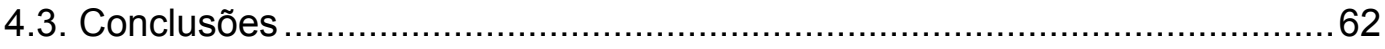

5. CONTROLE DE TENSÃO EM REDES ELÉTRICAS ..................................64

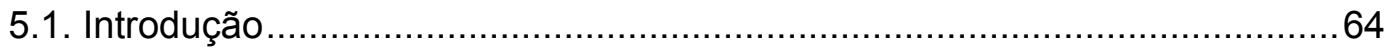

5.2. Motivações para o Controle de Tensão ......................................................65

5.3. Variação na Tensão pela Conexão de Geradores Eólicos ...........................66

5.4. Impactos da Integração de Turbinas Eólicas............................................68

5.5. Topologias de Turbinas Eólicas e Controle de Tensão [11] [12] ...................69

5.5.1. Controle de Potência da Turbina ..........................................................69

5.5.2. Turbina Eólica Com Velocidade Constante e Gerador de Indução ............70

5.5.3. Turbinas com Velocidade Variável ....................................................... 72

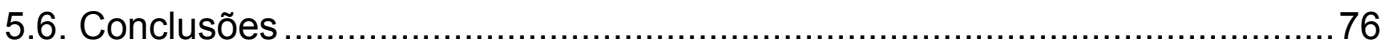

6. ESTUDO DE CASO - SISTEMA RADIAL DE 3 BARRAS ...........................78

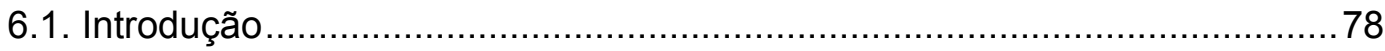

6.2. Conexão de Gerador sem Controle de Tensão.........................................78

6.3. Conexão de Gerador com Controle de Tensão .......................................... 82

6.4. Conexão de Compensador Síncrono na Barra de Carga .............................. 84 
6.4.1. Esgotamento da Capacidade de Controle de Tensão

6.4.2. Ponto de Operação na Parte Inferior da Curva $\phi$ Constante no

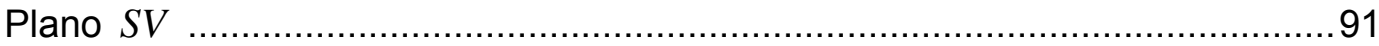

6.4.3. Atuação do Sistema de Controle do Compensador Síncrono ...................92 92

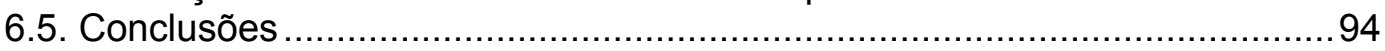

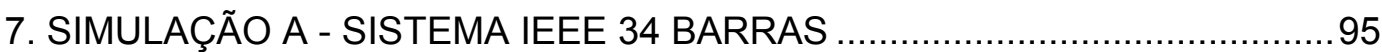

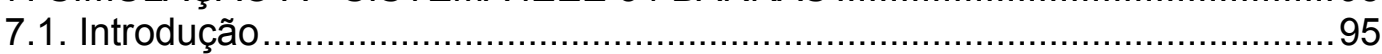

7.2. Simulação 1S - Inclusão de Gerador Distribuído na Barra 23 sem

Controle de Tensão e Carregamento do Sistema Constante ..............................98

7.3. Simulação 1C - Inclusão de Gerador Distribuído na Barra $23 \mathrm{com}$

Controle de Tensão e Carregamento do Sistema Constante...........................101

7.4. Simulação $2 S$ - Inclusão de Gerador Distribuído na Barra 23 sem

Controle de Tensão e Aumento do Carregamento do Sistema........................105

7.5. Simulação 2C - Inclusão de um Gerador Distribuído na Barra $23 \mathrm{com}$

Controle de Tensão e Aumento do Carregamento do Sistema .........................108

7.6. Avaliação do Impacto da Escolha da Tensão de Referência para

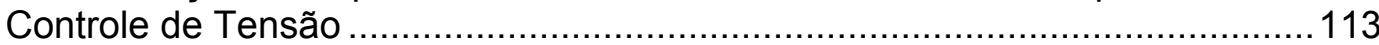

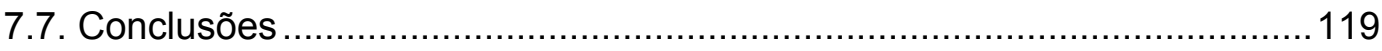

8. SIMULAÇÃO B - SISTEMA-TESTE DE 70 BARRAS ...................................122

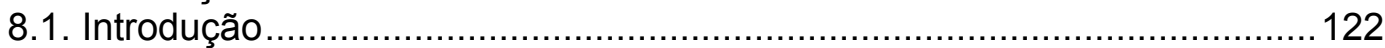

8.2. Simulação 1S - Inclusão de Gerador Distribuído na Barra 25 sem

Controle de Tensão e Carregamento do Sistema Constante...........................124

8.3. Simulação 1C - Inclusão de Gerador Distribuído na Barra $25 \mathrm{com}$

Controle de Tensão e Carregamento do Sistema Constante ...........................128

8.4. Simulação 2S - Inclusão de Gerador Distribuído na Barra 25 sem

Controle de Tensão e Aumento do Carregamento do Sistema........................131

8.5. Simulação 2C - Inclusão de Gerador Distribuído na Barra $25 \mathrm{com}$

Controle de Tensão e Aumento do Carregamento do Sistema.........................134

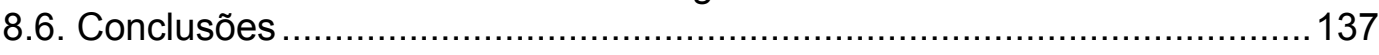

9. CONCLUSÕES E TRABALHOS FUTUROS ........................................... 140

10. REFERÊNCIAS BIBLIOGRÁFICAS .................................................. 143

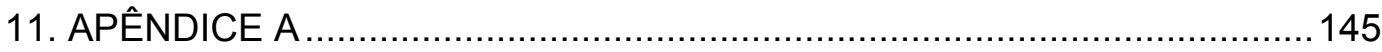




\section{Lista de figuras}

Figura 2.1 - Circuito de Duas Barras $\quad 21$

Figura 2.2 - Curvas no $\square^{3}$ de $P_{1}$ como Função de $V_{1}$ e $\theta_{1}$

Figura 2.3 - Curvas de $V_{1}$ Constante $(=0,9 \mathrm{pu}, 1,2 \mathrm{pu}, 1,5 \mathrm{pu}$ e $1,8 \mathrm{pu})$ no Plano $\theta_{1} P_{1}$

Figura 2.4 - $P_{1}$ Constante no Plano $\theta_{1} V_{1}$ e $Q_{1}$ Constante no Plano $\theta_{1} V_{1}$ para Diferentes Valores de $P_{1}$ e $Q_{1}$ em pu [5]

Figura 2.5 - Três Possibilidades de Solução para a Tensão na Carga com Mesmo Fator de Potência [1]

Figura 2.6 - Curva para Fator de Potência Constante na Barra de Carga no Plano PV [1]

Figura 2.7 - Circuito com as Impedâncias da Transmissão e da Carga [6]

Figura 2.8 - Limite de Estabilidade de Tensão sobre as Curvas de $\phi$ Constante no Plano PV [1]

Figura 2.9 - Sistema de Duas Barras com Capacitor na Barra Terminal

Figura 2.10 - Aumento e Diminuição da Tensão Respectivamente na Região Superior e Inferior da Curva com a Introdução de um Capacitor

Figura 2.11 - Diminuição da Tensão na Região Inferior da Curva com a Introdução de um Capacitor

Figura 2.12 - $\phi$ Constante e $Z_{c}$ Constante no Plano PV Com e Sem Capacitor

Figura 2.13 - Efeito da Introdução de Muitos Capacitores sobre a Tensão

Figura 2.14 - Curvas para $\phi$ Constante e para P Constante

Figura 3.1 - Potência Ativa e Reativa Saindo da Barra de Geração num Circuito de Duas Barras

Figura 3.2 - Gradientes $\nabla \mathrm{P}_{\mathrm{GL}}$ e $\nabla Q_{\mathrm{GL}}$ alinhados no Máximo Carregamento

Figura 3.3 - Localização do Ponto de Operação na Parte Superior da Curva SV

Figura 3.4 - Localização do Ponto de Operação na Parte Inferior da Curva SV 
Figura 4.1 - Localização do Vetor Gradiente de $P_{i}$ e $Q_{i}$ no Plano Ve

Figura 4.2 - Sinal da Margem na Curva SV

60

Figura 4.3 - Movimento dos Pontos de Operação Correspondendo à Deterioração da Margem

Figura 4.4 - Curva SV para Análise do Índice de Influência 62

Figura 5.1 - Sistema de 2 Barras com Geração Distribuída

Figura 5.2 - Configuração do Tipo Gerador de Indução com Rotor Gaiola de Esquilo

Figura 5.3 - Dependência da potência ativa e reativa da velocidade do rotor representada pelo escorregamento dada uma certa tensão terminal.

Figura 5.4 - Gerador de Indução Duplamente Alimentado

Figura 5.5 - Faixa de Operação do Gerador de Indução Duplamente Alimentado

Figura 5.6 - Curva de Capabilidade do Gerador Síncrono

Figura 6.1 - Diagrama Unifilar do Sistema de 3 Barras

Figura 6.2 - Curvas para Fator de Potência Constante para Fatores de Participação Diferentes sem Controle de Tensão na Barra 1

Figura 6.3 - Comparação entre as Curvas com Controle ( $V_{1}$ FIX) e sem Controle ( $V_{1}$ VAR) de Tensão na Barra 1 para Diferentes Fatores de Participação

Figura 6.4 - Sistema de 3 Barras com CS na Barra de Carga 85

Figura 6.5 - Curvas para a Barra 1

Figura 6.6 - Curva nos Planos $S_{2} V_{2}$ e $S_{2} Q_{g 2}$ (Configuração 0-100\%)

Figura 6.7 - Ponto A - Região Normal, Ponto B - Região Anormal e Ponto C - Fronteira entre as Regiões de Operação

Figura 6.8 - Perda do Controle de Tensão na Barra 2

Figura 6.9 - Atuação do Compensador Síncrono no Controle de Tensão da Barra 2

Figura 7.1 - Diagrama Unifilar do Sistema-Teste IEEE 34 Barras

Figura 7.2 - Fatores de Participação e Geração de Potência Ativa dos Geradores na Simulação $1 S$ 
Figura 7.4 - Perdas de Potência Ativa e Reativa do Sistema na Simulação 1S

Figura 7.5 - (a) Margem de Potência e (b) Ângulo $\beta$ por Barra e Caso de Carregamento

Figura 7.6 - Fatores de Participação e Geração de Potência Ativa dos Geradores na Simulação $1 \mathrm{C}$

Figura 7.7 - Perfil de Tensão do Sistema na Simulação 1C

Figura 7.8 - Perdas de Potência Ativa do Sistema na Simulação 1C

Figura 7.9 - (a) Margem de Potência e (b) Ângulo $\beta$ por Barra e Caso de Carregamento

Figura 7.10 - Perfil de Tensão do Sistema na Simulação 2S

Figura 7.11 - Perdas de Potência Ativa do Sistema na simulação 2S

Figura 7.12 - (a) Margem de Potência e (b) Ângulo $\beta$ por Barra e Caso de Carregamento

Figura 7.13 - Perfil de tensão do Sistema na Simulação 2C

Figura 7.14 - Perdas de Potência Ativa do Sistema na Simulação 2C

Figura 7.15 - (a) Margem de Potência e (b) Ângulo $\beta$ por Barra e Caso de Carregamento

Figura 7.16 - Perfil de Tensão do Sistema para Diferentes Valores de Tensão Terminal

Figura 7.17 - Máxima Carga para Diferentes Valores de Tensão Terminal

Figura 7.18 - Geração na Barra 23 para Diferentes Valores de Tensão Terminal

Figura 7.19 - Perdas de Potência Ativa para Diferentes Valores de Tensão Terminal

Figura 8.1 - Diagrama Unifilar do Sistema-Teste de 70 Barras

Figura 8.2 - Distribuição das Cargas pelo Sistema-Teste de 70 Barras

Figura 8.3 - Fatores de Participação e Geração de Potência Ativa e Reativa na Simulação $1 S$

Figura 8.4 - Perfil de Tensão do Sistema na Simulação 1S 
Figura 8.6 - (a) Margem de Potência e (b) Ângulo $\beta$ por Barra e Caso de Carregamento

Figura 8.7 - Fatores de Participação e Geração de Potência Ativa na Simulação 1C

Figura 8.8 - Perfil de Tensão do Sistema na Simulação 1C

Figura 8.9 - Perdas de Potência Ativa do Sistema na Simulação 1C

Figura 8.10 - (a) Margem de Potência e (b) Ângulo $\beta$ por Barra e Caso de Carregamento

Figura 8.11 - Perfil de Tensão do Sistema na Simulação 2S

Figura 8.12 - Perdas de Potência Ativa do Sistema na Simulação 2S

Figura 8.13 - (a) Margem de Potência e (b) Ângulo $\beta$ por Barra e Caso de Carregamento

Figura 8.14 - Perfil de Tensão do Sistema na Simulação 2C

Figura 8.15 - Perdas de Potência Ativa do Sistema na Simulação 2C

Figura 8.16 - (a) Margem de Potência e (b) Ângulo $\beta$ por Barra e Caso de Carregamento 


\section{Lista de tabelas}

Tabela 2.1 - Três Possibilidades de Solução para a Tensão na Carga com Mesmo Fator de Potência

Tabela 6.1 - Ponto de Operação do Caso-Base 79

Tabela 6.2 - Ponto de Operação no Máximo Carregamento 80

Tabela 6.3 - Índices de Estabilidade de Tensão das Barras 1 e 2

Tabela 6.4 - Ponto de Operação no Máximo Carregamento 82

Tabela 6.5 - Índices de Estabilidade de Tensão para as Barras 1 e 2 (V1 Controlado)

Tabela 6.6 - Ponto de Operação do Caso-Base 85

Tabela 6.7 - Pontos de Operação no Máximo Carregamento 86

Tabela 6.8 - Pontos A, B e C e suas Variações 89

Tabela 7.1 - Ponto de Operação do Caso-Base (Sistema-Teste 34 Barras)

Tabela 7.2 - Identificação das Simulações com o Sistema-Teste de 34 Barras

Tabela 7.3 - Casos de Carregamento da Simulação 2S 105

Tabela 7.4 - Casos de Carregamento da Simulação 2C 109

Tabela 7.5 - Comparação entre as Simulações 2S e 2C 112

Tabela 7.6 - Carga Máxima para Diferentes Valores de Tensão Terminal 116

Tabela 7.7 - Índices de Estabilidade de Tensão para a Barra 23

Tabela 8.1 - Ponto de Operação do Caso-Base (Sistema-Teste de 70 Barras)

Tabela 8.2 - Identificação das Simulações com o Sistema-Teste de 70 Barras

Tabela 8.3 - Síntese dos Resultados das Simulações no Sistema-Teste de 70 Barras 